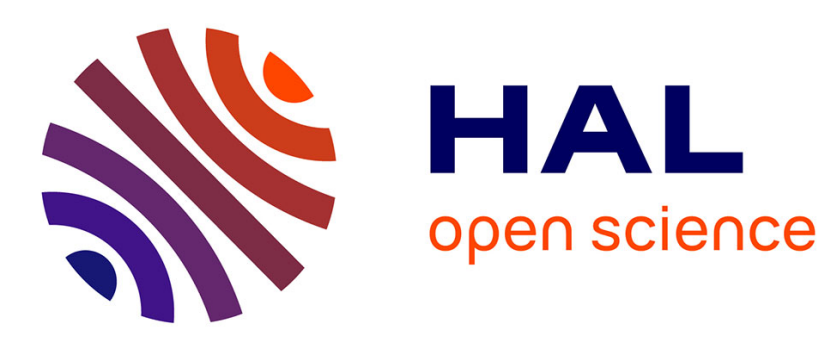

\title{
Strain estimation of moving tissue based on automatic motion compensation by ultrasound visual servoing
}

Pedro A Patlan-Rosales, Alexandre Krupa

\section{To cite this version:}

Pedro A Patlan-Rosales, Alexandre Krupa. Strain estimation of moving tissue based on automatic motion compensation by ultrasound visual servoing. IROS 2017 - IEEE/RSJ International Conference on Intelligent Robots and Systems, Sep 2017, Vancouver, Canada. pp. 2941-2946. hal-01572364

\section{HAL Id: hal-01572364 \\ https://hal.inria.fr/hal-01572364}

Submitted on 7 Aug 2017

HAL is a multi-disciplinary open access archive for the deposit and dissemination of scientific research documents, whether they are published or not. The documents may come from teaching and research institutions in France or abroad, or from public or private research centers.
L'archive ouverte pluridisciplinaire HAL, est destinée au dépôt et à la diffusion de documents scientifiques de niveau recherche, publiés ou non, émanant des établissements d'enseignement et de recherche français ou étrangers, des laboratoires publics ou privés. 


\title{
Strain estimation of moving tissue based on automatic motion compensation by ultrasound visual servoing
}

\author{
Pedro A. Patlan-Rosales ${ }^{1}$ and Alexandre Krupa ${ }^{2}$
}

\begin{abstract}
This paper presents a robot-assisted system to obtain elastic information of a moving tissue using a 2-D ultrasound probe actuated by a 6 degrees of freedom robotic arm. The proposed method combines ultrasound image-based visual servoing, force control and non-rigid motion estimation. We present how the motion estimation, while the force control and visual sevoing are enabled, is useful to compute the strain map of the tissue. Ex-vivo experiments performed on a moving abdominal phantom demonstrate the efficiency and robustness of this methodology to compute the strain information of a tissue in motion.
\end{abstract}

\section{INTRODUCTION}

One of the most frequently used medical imaging modality for patient examinations is ultrasound (US). It offers a wide variety of advantages such as portability, low cost and real-time image acquisition capability that is essential for robotized tele-echography [1]. Using the ultrasound, elastic information about the tissue can be computed by elastography. This process is performed by applying a mechanical compression with an ultrasound probe [2] or by measuring the propagation of a compressional wave (shear wave). The most common approach is based on mechanical compression of the tissue with the ultrasound probe. Elastography requires information acquired from the ultrasound transducer (also referred as ultrasound probe) as images (b-mode) or radiofrequency (RF) signals in at least two continuous states, preand post-compression. These two states are related to the external mechanical compression applied with the probe on the tissue and are used to estimate a strain map (elastogram). In a previous work, we proposed a palpation assistant system for ultrasound elastography that automatically moves a 2-D ultrasound probe to maintain the visibility of a stiff tissue of interest at the center of the field of view (FOV) [3]. In this previous work, a control system was proposed to combine three tasks: i) force control to generate the pre- and post-compression states required for the online elastogram estimation, ii) automatic centering of a stiff tissue target of interest that is tracked from the elastogram and iii) teleoperation of the orientation of the ultrasound probe in order to allow exploration of the stiff tissue targeted by the user. This system requires a processing step to segment and compute the centroid of the stiff tissue which is then automatically centered in the image by visual servoing.

\footnotetext{
${ }^{1}$ Pedro A. Patlan-Rosales is with Inria Rennes-Bretagne Atlantique, Universite de Rennes 1, campus universitaire de Beaulieu, Rennes 35042, France. pedro-alfonso.patlan-rosalesdinria.fr

${ }^{2}$ Alexandre Krupa is with Inria Rennes-Bretagne Atlantique, campus universitaire de Beaulieu, Rennes 35042, France. alexandre.krupa@inria.fr
}

The proposed approach showed good performance under small perturbations of the system, since it was designed for motionless tissues. However, this approach failled in case of a moving tissue since perturbation motion generates large noises in the estimated elastogram. In this paper, our main objective is therefore to provide a solution that allows the strain map estimation of a moving tissue that was not possible with our previous work [3]. To address this issue, we propose a new robotic solution for elastogram estimation from b-mode images provided by a 2-D ultrasound probe. It is based on the use of a tissue deformation tracking algorithm and an automatic 6 degrees of freedom (DOF) compensation of the perturbation motion by ultrasound visual servoing using dense information.

Tracking deformable tissues has been studied in several works. For example, in heart surgery, the surface of a beating heart was tracked in stereoscopic images using a thin-plate spline deformation model [4]. Similar methodology was presented to track the deformation applied to a soft tissue phantom from 3D ultrasound images [5]. In this latter work a 3-D non-rigid tracking algorithm based on a thin-plate spline 3-D deformation model was considered and a visual servoing scheme was designed to automatically move the ultrasound probe to compensate the rigid motion components of the tissue. Recently, an approach based on a mass-spring-damper model combined with the dense information contained in a sequence of ultrasound 3D images was proposed to track the deformation of soft tissues [6].

Very few investigations have been undertaken regarding the use of ultrasound elastography in robot-assisted procedures. These works are related to the field of laparoscopy. For example, a snake-like robot was presented in [7], where a micro ultrasound probe attached at the distal part of the robot was used to find hard lesions by palpation motion. The da Vinci surgical robot (Intuitive Surgical Inc.) was also used to obtain elastic information of a tissue of interest by controlling the motion of a laparoscopic 2-D ultrasound probe [8]. Using a similar framework, in [9] a mechanical vibrator placed on the skin of the patient was proposed instead of controlling the motion of the ultrasound probe. However, none of these works were applied to obtain the strain information of a moving tissue which is a challenging issue.

In this paper we propose to exploit the capabilities of non-rigid motion estimation in 2-D ultrasound images to estimate the displacement map required to compute the tissue strain map. For this, we consider the intensity changes in the ultrasound images due to the force applied by the probe. Moreover, in order to allow strain map estimation of a 
moving tissue, we propose in our approach to perform an automatic motion compensation using an ultrasound imagebased visual servoing that synchronizes the probe and tissue motions during the strain map estimation process.

The proposed approach is detailed in section II. We present first the non-rigid motion estimation of the soft tissue, then the robot control based on ultrasound visual servoing that allows the probe motion synchronization with moving soft tissue, and the process to obtain the elastogram. In section III, we present and discuss the results obtained from first ex-vivo experiments performed on an abdominal phantom.

\section{MethoD}

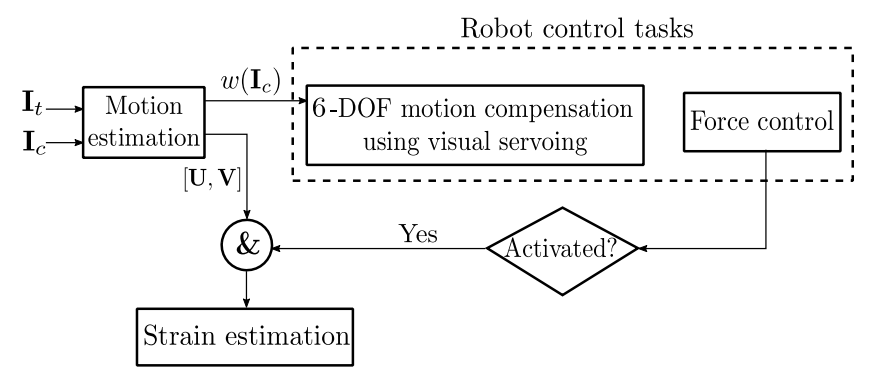

Fig. 1: Proposed methodology to obtain the strain map of a moving tissue. This diagram shows the steps to estimate the elastogram for two ROIs in the ultrasound images $\mathbf{I}_{t}$ and $\mathbf{I}_{c}$. These images are the reference and current images respectively. $w\left(\mathbf{I}_{c}\right)$ represents the image $\mathbf{I}_{c}$ with a nonrigid transformation $(w)$ applied in the motion estimation to reduce the absolute difference with $\mathbf{I}_{t}$. $[\mathbf{U}, \mathbf{V}]$ are the lateral and axial displacement maps computed between $\mathbf{I}_{c}$ and $\mathbf{I}_{t}$ after the motion estimation. Symbol \& indicates a logic AND operator.

In this section, we propose a system to estimate the elastogram of a moving tissue. Fig 1 shows the workflow of our proposed method. The main problem produced by a moving object is the generation of noises in the elastogram, due to the introduction of non-axial motion. Our system combines ultrasound dense visual servoing, force control and non-rigid motion estimation to compute the elastogram of moving tissue. In the next subsections we describe every block of the methodology featured in Fig 1.

\section{A. Motion estimation of a deformable tissue}

Due to the nature of soft tissue to be highly deformable, we propose to use a dense non-rigid motion estimation in 2$\mathrm{D}$ to track the soft tissue deformation in a region of interest (ROI) of the 2D image under mechanical compression. The goal of this process is to estimate the deformation parameters $\mathbf{p}$ of an initial image template (ROI) $I_{t} \in \mathbb{R}^{m \times n}$ composed of $N$ pixels relative to a region extracted from the current image $I_{c}$ that is transformed with a warp function $w$ :

$$
\hat{\mathbf{p}}=\arg \min _{\mathbf{p}} \sum_{k=0}^{N-1} E\left(I_{t}\left(\mathbf{x}_{k}\right), I_{c}\left(w\left(\mathbf{x}_{k}, \mathbf{p}\right)\right)\right)
$$

where $w(\mathbf{x}, \mathbf{p})$ warps the image point $\mathbf{x}$ using the parameters $\mathbf{p}$ and $E$ is a dissimilarity function.

In many image processing cases the sum of squared differences (SSD) of the pixel intensities is used as function $E$ due to the low computational cost. The SSD lacks of robustness to the change in image intensity which is a common occurrence when the ultrasound probe is moving. Therefore, we propose to use the sum of conditional variance (SCV) [10] which is robust to global illumination changes, and it is expressed as follows:

$$
S C V(\mathbf{p})=\sum_{k=0}^{N-1}\left[\hat{I}_{t}\left(\mathbf{x}_{k}\right)-I_{c}\left(w\left(\mathbf{x}_{k}, \mathbf{p}\right)\right)\right]^{2}
$$

where $\hat{I}_{t}$ is the image intensity adaptation of the template relative to the image intensity conditions in the current warped image $I_{c}(w(\mathbf{x}, \mathbf{p}))$. The image template adaptation is performed through the expectation operator $\mathcal{E}$ as $\hat{I}_{t}=$ $\mathcal{E}\left(I_{c}(w(\mathbf{x}, \mathbf{p})) \mid I_{t}(\mathbf{x})\right)$. Thus, the adaptation of every gray level for the reference $I_{t}$ is,

$$
\hat{I}_{t}(\mathbf{x})=\sum_{i=0}^{L-1} i \frac{p_{I_{t} I_{c}}(i, j)}{p_{I_{t}}(j)}
$$

where $\mathrm{L}$ is the gray level for the template image $I_{t}$ and current image $I_{c} \cdot p_{I_{t}}$ is the probability density function of $I_{t}$ and $p_{I_{t} I_{c}}$ is the joint probability density function of $I_{t}$ and $I_{c}$. These functions are computed as follows:

$$
\begin{aligned}
p_{I_{t} I_{c}}(i, j) & =p_{I_{t} I_{c}}\left(I_{c}(w(\mathbf{x}, \mathbf{p}))=i, I_{t}(\mathbf{x})=j\right) \\
& =\frac{1}{N} \sum_{k=0}^{N-1} \delta\left(I_{c}\left(w\left(\mathbf{x}_{k}, \mathbf{p}\right)\right)-i\right) \delta\left(I_{t}\left(\mathbf{x}_{k}\right)-j\right) \\
p_{I_{t}}(i) & =\sum_{i=0}^{L-1} p_{I_{t} I_{c}}(i, j)
\end{aligned}
$$

where $\delta$ is a Dirac delta function such as $\delta(u)=1 \Leftrightarrow u=0$. We solved this non-linear optimization by using the inverse scheme presented in [11]. In this case the aim is to align the template $I_{t}$ on a transformation of the current image $I_{c}$ using the increment of warp parameter ${\widehat{\Delta \mathbf{p}_{l}}}_{l}$ as:

$$
\widehat{\Delta \mathbf{p}_{l}}=\arg \min _{\Delta \mathbf{p}_{l}} \sum_{k=0}^{N-1}\left[\hat{I}_{t}\left(w\left(\mathbf{x}_{k}, \Delta \mathbf{p}_{l}\right)\right)-I_{c_{l}}\left(w\left(\mathbf{x}_{k}, \mathbf{p}_{l}\right)\right)\right]^{2}
$$

where $l$ is the iteration number of the iterative optimization process and the update rule is expressed as:

$$
w\left(\mathbf{x}, \mathbf{p}_{l+1}\right)=w\left(w^{-1}\left(\mathbf{x}, \Delta \mathbf{p}_{l}\right), \mathbf{p}_{l}\right)
$$

This iterative process ends when $S C V$ is minimum or when $l$ has reached $N_{i t}$ (maximum number of iterations). The optimization of (6) is then expressed as:

$$
{\widehat{\Delta \mathbf{p}_{l}}}_{l}=-\mathbf{J}^{+}(\Delta \mathbf{p})\left[\hat{\mathbf{I}}_{t}-w\left(\mathbf{I}_{c_{l}}, \mathbf{p}\right)\right]
$$

where $\mathbf{J}(\Delta \mathbf{p})$ is the Jacobian matrix associated to $\operatorname{SCV}(\Delta \mathbf{p})$ and + is the Moore-Penrose pseudo-inverse matrix. $\hat{\mathbf{I}}_{t}$ and $\mathbf{I}_{c_{l}}$ are the vectors containing every pixel in the images $\hat{I}_{t}$ and $I_{c_{l}}$ respectively. The Jacobian $\mathbf{J}(\Delta \mathbf{p})$ can be defined as:

$$
\mathbf{J}(\Delta \mathbf{p})=\nabla I_{c} \frac{\partial w}{\partial \Delta \mathbf{p}}
$$

where $\nabla I_{c}$ is the gradient of the image $I_{c}$.

Deformable motion estimation depends on the selection of 
the warping function $w$. We chose the thin-plate splines (TPS) function which has been used for dense image tracking in [12] and [13]. TPS warping function is a combination of an affine transformation and deformation parameters as follows:

$$
w(\mathbf{x}, \mathbf{p})=\left(\begin{array}{ll}
a_{0} & a_{1} \\
a_{3} & a_{4}
\end{array}\right) \mathbf{x}+\left(\begin{array}{l}
a_{2} \\
a_{5}
\end{array}\right)+\sum_{k=0}^{N_{c}-1}\left(\begin{array}{c}
\kappa_{x}^{k} \\
\kappa_{y}^{k}
\end{array}\right) \phi\left(d\left(\mathbf{x}, \mathbf{c}_{k}\right)\right)
$$

where $N_{c}$ is the number of control points $\mathbf{c}, \kappa_{x}^{k}$ and $\kappa_{y}^{k}$ are the weights of each $k$ control point along the $x$ and $y$ axes respectively. $\phi$ is the thin-plate kernel defined as $\phi(x)=$ $x^{2} \frac{\log (x)}{2}$ and $d(\mathbf{x}, \mathbf{y})$ is the euclidean distance between the points $\mathbf{x}$ and $\mathbf{y}$. The parameter vector of the warping function p of dimension $2 N_{c}+6$ is expressed as:

$$
\mathbf{p}^{\top}=\left(\begin{array}{llllllll}
a_{0} & a_{1} & a_{2} & a_{3} & a_{4} & a_{5} & \boldsymbol{\kappa}_{x}^{\top} & \boldsymbol{\kappa}_{y}^{\top}
\end{array}\right)
$$

where the first six parameters are the parameters of the affine transformation. $\boldsymbol{\kappa}_{x}$ and $\boldsymbol{\kappa}_{y}$ are vectors $\left(N_{c}\right.$ elements) containing the weights $\kappa_{x}^{k}$ and $\kappa_{y}^{k}$ respectively.

Once we selected the ROI, we define the control points position $\mathbf{c}$ inside the ROI that are initially distributed in an equidistant grid. For more details about the warping function $w$ refer to [12], [13]. The optimization requires the Jacobian of $w$ which can be obtained as:

$$
\frac{\partial w}{\partial \Delta \mathbf{p}}=\left(\begin{array}{ll}
\mathbf{J}_{\mathbf{A}} & \mathbf{J}_{\boldsymbol{\kappa}}
\end{array}\right)
$$

where:

$$
\begin{aligned}
\mathbf{J}_{\mathbf{A}} & =\left(\begin{array}{cccccc}
x & y & 1 & 0 & 0 & 0 \\
0 & 0 & 0 & x & y & 1
\end{array}\right) \in \mathbb{R}^{2 \times 6}, \\
\mathbf{J}_{\boldsymbol{\kappa}} & =\left(\begin{array}{cccccc}
\boldsymbol{\phi} & & 0 & \cdots & 0 \\
0 & \cdots & 0 & & \boldsymbol{\phi}
\end{array}\right) \in \mathbb{R}^{2 \times 2 N_{c}}, \\
\boldsymbol{\phi} & =\left(\begin{array}{lll}
\phi\left(d\left(\mathbf{x}, \mathbf{c}_{0}\right)\right) & \cdots & \left.\phi\left(d\left(\mathbf{x}, \mathbf{c}_{N_{c}-1}\right)\right)\right)
\end{array}\right.
\end{aligned}
$$

$x$ and $y$ are the pixel coordinates in the image $I_{t}$.

We have described the motion estimation process we implemented in our system. However, we need to maintain the position of the ROI stable even when the tissue is moving. This can cause motions not only in-plane but also out-ofplane as emphasized in [14] (see Fig. 2a). This tissue motion can be compensated by controlling the motion of a 2-D US probe using the intensity-based ultrasound visual servoing presented in [15].

\section{B. 6-DOF motion compensation by dense visual servoing}

We propose to use a similar visual servoing approach to the one presented in [15] to control a robotic arm holding the probe in order to automatically compensate the relative non-axial motions between the probe and the moving tissue of interest to analyse. These non-axial motions correspond to one lateral and one rotational motions in the US plane and one lateral and two rotational motions out of the US plane (see Fig. 2a).

In [15], a visual servoing method that uses the intensity information of the pixels inside a ROI has demonstrated the feasibility to control the $6 \mathrm{DOF}$ of a 2-D ultrasound probe for compensating both in-plane and out-of-plane rigid motions.
However, in this previous work the tissue was assumed rigid without considering deformation due to internal physiological motion or the presence of mechanical compression, which is our case during the elastogram estimation process. Therefore, we propose to improve the method of [15] by using the non-rigid motion estimation algorithm presented in section II.A to deal with the soft tissue deformations. We briefly recall the principle of the ultrasound dense visual

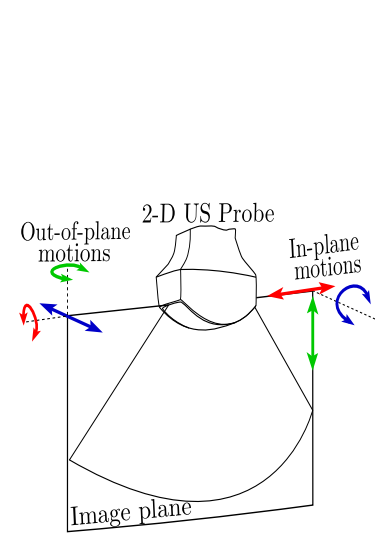

(a)

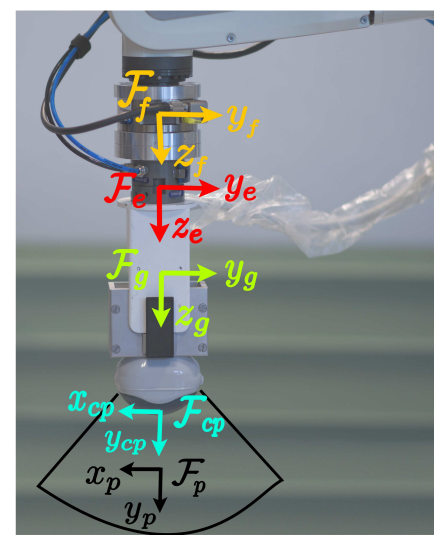

(b)
Fig. 2: (a) Possible motions with a 2-D US probe. (b) Cartesian reference frames attached to the 6-DOF robotic arm.

servoing approach [15]. The aim is to control the probe velocity $\mathbf{v}=\left[\begin{array}{llllll}v_{x} & v_{y} & v_{z} & \omega_{x} & \omega_{y} & \omega_{z}\end{array}\right]^{\top}$ where the three first elements correspond to the translational velocity and the three last elements to the angular velocity expressed in the frame $\mathcal{F}_{c p}$ (see Fig. 2b). The visual features vector, s, used for this scheme contains the intensities of the pixels inside a ROI such as:

$$
\mathbf{s}_{I}=\left(I_{1,1}, \ldots, I_{m, n}\right)
$$

where $I_{u, v}$ is the intensity in gray level for the 2-D pixel coordinates $(u, v)$ in the US image. The interaction matrix that relates the variation of the pixel intensity to the probe velocity $\mathbf{v}$, such that $I_{u, v}=L_{I_{u, v}} \mathbf{v}$, is given by:

$$
L_{I_{u, v}}=\left[\begin{array}{lllll}
\nabla I_{x} & \nabla I_{y} & \nabla I_{z} & y \nabla I_{z}-x \nabla I_{z}\left(x \nabla I_{y}-y \nabla I_{x}\right)
\end{array}\right]
$$

where $\nabla I_{u, v}=\left[\begin{array}{lll}\nabla I_{x} & \nabla I_{y} & \nabla I_{z}\end{array}\right]$ corresponds to the 3-D image gradient associated with the pixel $(u, v)$. The three components, $\nabla I_{x}=\frac{\partial I_{u, v}}{\partial x}, \nabla I_{y}=\frac{\partial I_{u, v}}{\partial y}$ and $\nabla I_{z}=\frac{\partial I_{u, v}}{\partial z}$ are obtained with 3-D derivative filters, as performed in [15], applied to a thin volume composed of 5 parallel slices captured by moving the probe during an initial procedure before launching the visual servoing. The values of $x$ and $y$ are the metric coordinates of the pixel $(u, v)$ in the image obtained from the intrinsic parameters of the probe:

$$
\left(\begin{array}{l}
x \\
y
\end{array}\right)=\left(\begin{array}{c}
s_{x}\left(u-u_{c p}\right) \\
s_{y}\left(v-v_{c p}\right)
\end{array}\right)
$$

where $\left(s_{x}, s_{y}\right)$ are the pixel width and height and $\left(u_{c p}, v_{c p}\right)$ are the pixel coordinates of the origin of the contact point frame, $\mathcal{F}_{c p}$, in the ultrasound 2-D image. The definition of the interaction matrix in (14) is related to one pixel. The interaction matrix $\mathbf{L}_{s} \in \mathbb{R}^{m n \times 6}$ that associates the intensity 
variation of all the pixels in the ROI (visual features) to the probe velocity $\mathbf{v}$ is then obtained by stacking all the $m \times n$ interaction matrices $L_{I_{u, v}}$ for every pixel as:

$$
\mathbf{L}_{s}=\left(\begin{array}{lll}
L_{I_{1,1}} & \ldots & L_{I_{m, n}}
\end{array}\right)^{\top}
$$

In order to automatically compensate the moving tissue, we define the visual error as $\mathbf{e}_{s}=\mathbf{s}_{w I}-\mathbf{s}_{I}^{*}$, and we establish the desired visual error variation as $\dot{\mathbf{e}}_{s}^{*}=-\lambda_{s} \mathbf{e}_{s}$ with $\lambda_{s}$ being the visual control gain. Unlike [15], here $\mathbf{s}_{w I}=I_{c}(w(\mathbf{x}, \mathbf{p}))$ is the current image warped with the TPS warping function using the current parameters $\mathbf{p}$. This major improvement allows the visual servoing approach to be robust to the presence of the non-rigid motion induced by the tissue deformation. The desired pixel intensities vector is $\mathbf{s}_{I}^{*}=\left(I_{t_{1,1}}, \ldots, I_{t_{m, n}}\right)$. Then, the control law for the motion of the probe is provided by:

$$
\mathbf{v}_{s}=\mathbf{L}_{s}^{+} \dot{\mathbf{e}}_{s}^{*} .
$$

\section{Force control}

In order to obtain the pre- and post-compression states of the tissue, we propose to apply a varying force along the axial direction of the ultrasound probe at the contact frame $\mathcal{F}_{c p}$ (see Fig 2b) by using a force control scheme. The robotic arm that is used in our work is equipped with a 6-axes force/torque sensor providing a tensor measurement ${ }^{f} \mathbf{H}_{f}$ in the force sensor frame $\mathcal{F}_{f}$. To measure the interaction force between the probe and the tissue expressed in the contact frame $\mathcal{F}_{c p}$, we have to consider the probe mass $m_{p}$ in order to compensate the gravity force tensor ${ }^{g} \mathbf{H}_{g}=$ $\left[\begin{array}{llllll}0 & 0 & 9.81 m_{p} & 0 & 0 & 0\end{array}\right]^{\top}$. The force tensor applied to the tissue can then be expressed in the frame $\mathcal{F}_{c p}$ as follows:

$$
{ }^{c p} \mathbf{H}_{c p}={ }^{c p} \mathbf{F}_{f}\left({ }^{f} \mathbf{H}_{f}-{ }^{f} \mathbf{F}_{g}{ }^{g} \mathbf{H}_{g}\right)
$$

where ${ }^{f} \mathbf{F}_{g}$ and ${ }^{c p} \mathbf{F}_{f}$ are transformation matrices from the gravity frame $\mathcal{F}_{g}$ to the frame $\mathcal{F}_{f}$ and from the frame $\mathcal{F}_{f}$ to the frame $\mathcal{F}_{c p}$ respectively.

Since our goal is to control only the force component along the $y$-axis (axial direction) of the probe, we define the feature vector to be regulated as $\mathbf{s}_{f}=$ $\left[\begin{array}{llllll}0 & 1 & 0 & 0 & 0 & 0\end{array}\right]^{c p} \mathbf{H}_{c p}$. In order to apply a continuous compression motion, we propose to apply the following desired varying force that is based on a sinusoidal function:

$$
F_{d}(\tau)=\frac{\Delta_{F}}{2}\left[\sin \left(\frac{(4 \tau-T) \pi}{2 T}\right)+1\right]+F_{0},
$$

where $\tau$ is the discrete sample iteration number and $\Delta_{F}$ is the amplitude of the sinusoidal function. $T$ is the period of the curve expressed in sample time and $F_{0}$ is the initial force. Then, the desired feature vector can be defined as $\mathbf{s}_{f}^{*}=\left[\begin{array}{llllll}0 & F_{d}(\tau) & 0 & 0 & 0 & 0\end{array}\right]$, and the error to minimize is $\mathbf{e}_{f}=\mathbf{s}_{f}-\mathbf{s}_{f}^{*}$, with an exponential decrease of $\mathbf{e}_{f}$.

The desired variation of the error is defined as $\dot{\mathbf{e}}_{f}^{*}=$ $-\lambda_{f} \mathbf{e}_{f}$ with $\lambda_{f}$ being the force control gain. The change of the force feature through time can be expressed as $\dot{\mathbf{s}}_{f}=\mathbf{L}_{f} \mathbf{v}$, where $\mathbf{L}_{f}=\left[\begin{array}{llllll}0 & K & 0 & 0 & 0 & 0\end{array}\right]$ is an approximation of the interaction matrix related to the force feature with $K$ being an estimation of the contact stiffness between the probe and the tissue. Therefore, we define the control law for the oscillatory motion of the ultrasound probe along the y-axis as:

$$
\mathbf{v}_{f}=\mathbf{L}_{f}^{+} \dot{\mathbf{e}}_{f}^{*}
$$

\section{Control fusion}

In order to fuse the automatic motion compensation by visual servoing and the force control, we can define a control law for the probe velocity $\mathbf{v}$ using the redundancy control framework [16]. We use the force control law as the highest priority task remaining as in equation (17). Then, the secondary task, visual servoing, is expressed as:

$$
\mathbf{v}_{s}=\left(\mathbf{L}_{s} \mathbf{P}_{f}\right)^{+}\left(\dot{\mathbf{e}}_{s}^{*}-\mathbf{L}_{s} \mathbf{v}_{f}\right)
$$

where $\mathbf{P}_{f}=\mathbf{I}-\mathbf{L}_{f}^{+} \mathbf{L}_{f}$ is the projector operator onto the null space of $\mathbf{L}_{f}$. $\mathbf{I}$ is the identity matrix of size 6 .

Finally, the general control law that allows to control the 6-DOF of the probe is given from (20) and (21) as:

$$
\mathbf{v}=\mathbf{v}_{f}+\mathbf{v}_{s}
$$

\section{E. Strain estimation}

Strain estimation is a process that depends of the motion estimation of the elements contained in the ROI to obtain an elastogram. From the non-rigid motion estimation presented in section II.A, we can obtain the displacement maps $U(x, y)$ and $V(x, y)$ (lateral and axial directions respectively). Let define $\mathbf{x}^{\prime}=w(\mathbf{x}, \mathbf{p})$ as the corresponding coordinate of $\mathbf{x}$ after the tissue deformation. For every $\mathbf{x}$ we have a displacement vector $D(\mathbf{x})=\mathbf{x}^{\prime}-\mathbf{x}$. Then, we can obtain $U(\mathbf{x})$ and $V(\mathbf{x})$ as the lateral and axial components of the displacement vector $D(\mathbf{x})$ (see Fig. 3).

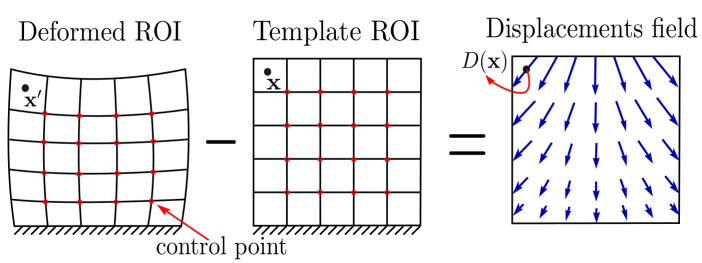

Fig. 3: Displacement map obtained from the deformation of the ROI. $\mathbf{x}$ and $\mathbf{x}^{\prime}$ represent respectively the coordinates of one pixel in $I_{t}$ and its new coordinates in the current image $I_{c}$ after tissue deformation. $D(\mathbf{x})$ is the vector displacement from $\mathbf{x}$ to $\mathbf{x}^{\prime}$.

Assuming that we have at least a grid of $3 \times 3$ control points, then the elastogram $\varepsilon(x, y)$ can be computed as in [3] by convolving a least-squared (LSQ) strain filter with the axial displacement map $V(x, y)$. We chose the strain filter presented in [17] that is defined as:

$$
g(k)=\xi(k)\left[\begin{array}{ll}
1 & -\frac{k+1}{2}
\end{array}\right]\left[\begin{array}{cccc}
1 & 2 & \ldots & k \\
1 & 1 & \ldots & 1
\end{array}\right]
$$

where $\xi(k)=\frac{12}{k\left(k^{2}-1\right)}$ and $k$ is the number of elements of the filter.

The elastogram $\varepsilon(x, y)$ is the output of the proposed system. We present in the next section the obtained experimental results. 


\section{EXPERIMENTAL RESULTS}

We perform experiments using a 6-DOF robot Viper s850 (Adept Technology Inc., USA) equipped with a force/torque sensor ATI Gamma 65-SI FT connected to a National Instrument NI DAQmx PCI-6220 board. We also attached to the robot end-effector a convex ultrasound probe (Analogic Corporation 4DC7-3/40) in 2-D mode. The ultrasound probe was plugged in a SonixTouch ultrasound scanner, which was sending the images to a client running on a workstation (Intel Xeon CPU @ 2.1 GHz) that performs all the imaging process, control law computation and communication with the robot. The images from the scanner were sent to the workstation at the rate of $\sim 40$ FPS (frames per second). Force control was performed at higher frequency $(200 \mathrm{~Hz})$. We developed a C++ software with a graphical user interface (GUI), and we used ViSP [18] for the communication with the robot.

The experiments were performed on an ABDFAN ultrasound phantom (Kyoto Kagaku Co., Japan) simulating the abdomen of a body. Fig. 5a shows a picture of the experimental setup. Now, we describe the complete process for one experiment. Initially, the probe was positioned above the phantom without contact. Then, through the GUI, we enabled the force control without oscillation $\left(F_{0}=5 \mathrm{~N}\right.$ and $\Delta_{F}=0 \mathrm{~N}$ ), and we can see that the measured force value reaches the desired force in the strip (light gray background) of the plot in the Fig. 4c.

Once we have selected a ROI in the ultrasound image, a grid of $5 \times 5$ control points is placed inside the ROI as shown in Fig 5e, and the oscillatory force variation is activated $\left(\Delta_{F}=2 \mathrm{~N}\right)$ with the soft tissue motion estimation process. The strain map is estimated and displayed in real time (see Fig. 5i). Then, a thin volume composed of five parallel ultrasound images is acquired to obtain the 3D image gradient (during the period of time with light gray background in Fig 4a) just before launching the automatic soft tissue motion compensation by visual servoing. The curves in the Fig. 4 show the evolution through time of the experiment, where we manually apply to the abdominal phantom small and large motions to test the robustness of the system to perturbation motions. Some states during the temporal evolution of the experiment are shown in Fig. 5, where we can observe in the first row (images (a) to (d)) the kind of manual motion applied to the phantom that was automatically compensated by the visual servoing. In the same figure, we show for every state the corresponding bmode image with the grid of control points (images (e) to (h)) and the resulting strain maps (images (i) to (l)). The color representation of the strain maps indicates in red soft tissue and in white the hard tissue. We can easily observe in the brightest region of the obtained strain maps that a hard tissue target corresponding to an artificial cyst always remains visible in the center of the elastogram even when the phantom is moving.

Fig. 6 shows three elastograms obtained under different conditions. In Fig. 6a, the strain map has been estimated using only oscillatory force control without the automatic

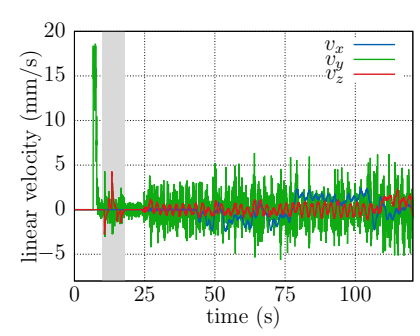

(a) Probe linear velocities

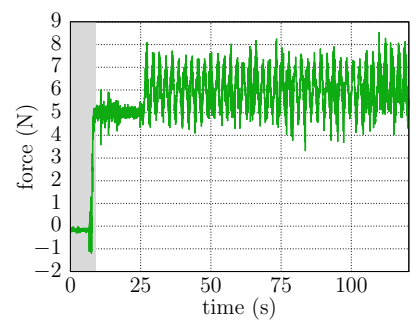

(c) Force

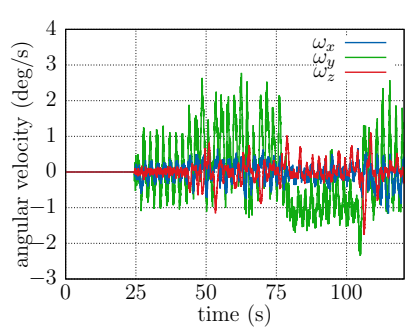

(b) Probe angular velocities

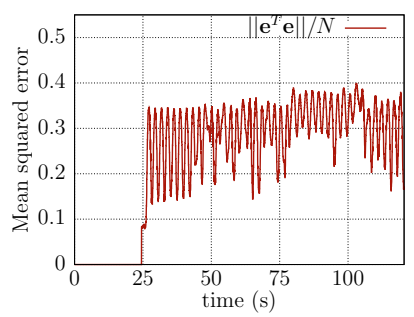

(d) Visual error
Fig. 4: Evolution through time of the reference experiment. (a) to (d) show the curves of the velocities, measured force and visual error during the experiment.

motion compensation when the phantom was motionless. We can see that the cyst is very visible as opposed to the elastogram presented in Fig. 6b where a perturbation motion was applied to the phantom without activating the automatic compensation by visual servoing. It is clear that in this case the elastogram estimation is perturbed and can not provide any useful information. Fig. $6 \mathrm{c}$ shows the obtained elastogram when the phantom is moving and the automatic motion compensation by visual servoing is activated. This last test demonstrates the efficiency of our approach since the cyst is very visible and similar to the case where the phantom was motionless.

\section{Conclusion}

We presented a new approach to obtain the elastogram of a moving tissue. Our method is based on three main parts. First, a robust non-rigid motion estimation based on dense information that allows the tracking of a ROI under compression. Second, a dense visual servoing approach that automatically moves the ultrasound probe to compensate the in-plane and out-of-plane motion of a moving tissue. Third, a force control that applies the oscillatory compression required for the strain estimation process. Preliminary exvivo results have demonstrated the feasibility to estimate the strain map of a moving tissue. Future work will consist in validating this new elastography approach with in-vivo conditions.

\section{ACKNOWLEDGMENT}

This work has been supported by Inria Rennes - Bretagne Atlantique and the National Council of Science and Technology of Mexico (CONACYT) under the grant no. $242120 / 383765$. This work was also partly funded by the French program "Investissement d'Avenir" run by the 'Agence Nationale pour la Recherche'; the grant reference is 


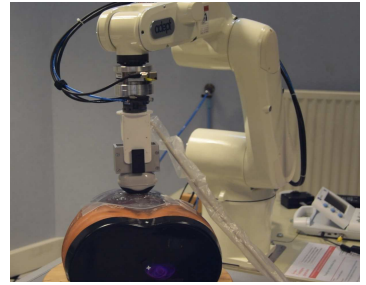

(a) Initial state
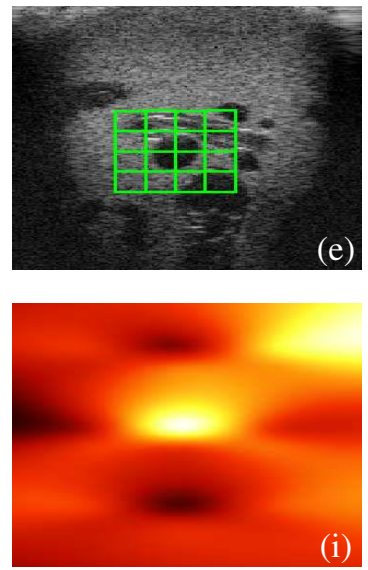
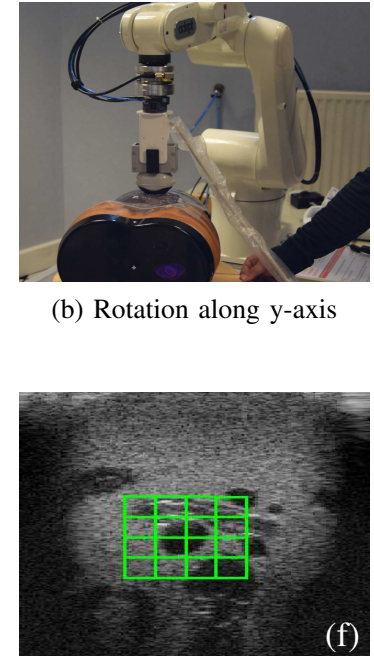

(b) Rotation along y-axis

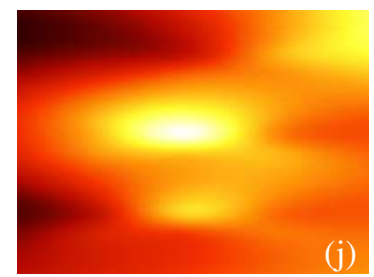

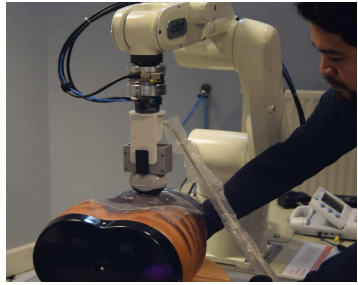

(c) Perturbation at the bottom of the phantom
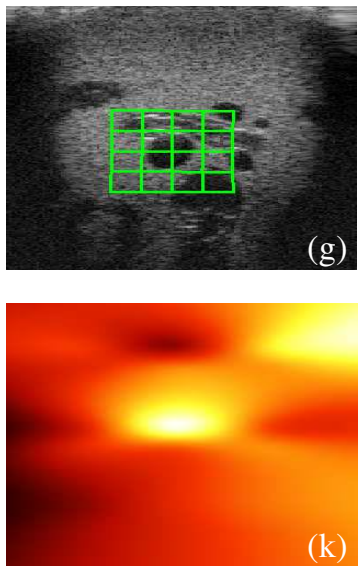

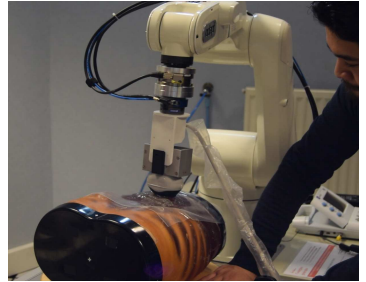

(d) Perturbation at one side of the phantom
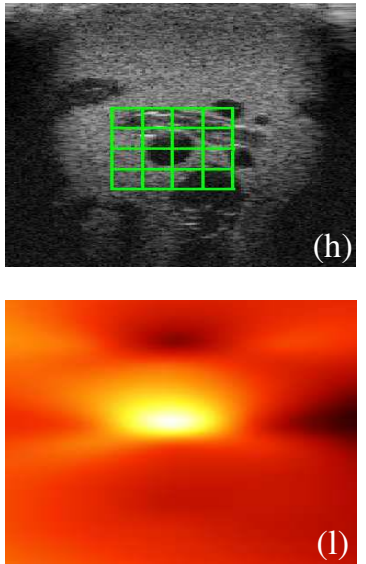

Fig. 5: Perturbations introduced to the phantom. (a) to (d) show some of the states during the experiment. (e) to (h) show the b-mode images with the current control positions. (i) to (1) show the strain maps for every state.
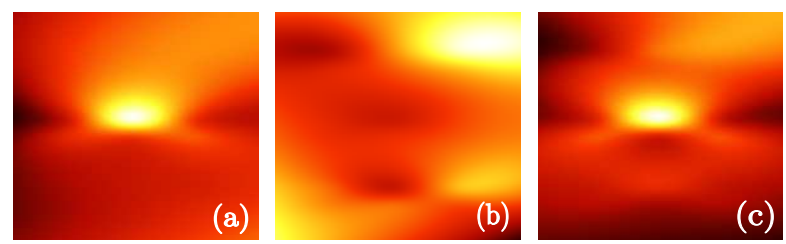

Fig. 6: Strain maps obtained under the following conditions: (a) Oscillatory force control active, no perturbation motion applied. (b) Out-of-plane perturbation motion applied and motion compensation disabled. (c) Outof-plane perturbation motion applied and motion compensation activated.

'Infrastructure d'avenir en Biologie Santé - ANR-11-INBS0006'.

\section{REFERENCES}

[1] A. Krupa, D. Folio, C. Novales, P. Vieyres, and T. Li, "Robotized teleechography: an assisting visibility tool to support expert diagnostic," IEEE Systems Journal, no. 3, pp. 974-983, Sep 2016.

[2] J. Ophir, "Elastography: A quantitative method for imaging the elasticity of biological tissues," Ultrasonic Imaging, vol. 13, no. 2, pp. 111-134, Apr. 1991

[3] P. A. Patlan-Rosales and A. Krupa, "Automatic palpation for quantitative ultrasound elastography by visual servoing and force control," in IEEE/RSJ Int. Conf. on Intelligent Robots and Systems, Daejeon, 2016.

[4] R. Richa, P. Poignet, and C. Liu, "Three-dimensional motion tracking for beating heart surgery using a thin-plate spline deformable model," Int. J. Rob. Res., vol. 29, no. 2-3, pp. 218-230, Feb 2010.

[5] D. Lee and A. Krupa, "Intensity-based visual servoing for non-rigid motion compensation of soft tissue structures due to physiological motion using 4d ultrasound," in IEEE/RSJ Int. Conf. on Intelligent Robots and Systems, San Francisco, 2011, pp. 2831-2836.

[6] L. Royer, A. Krupa, G. Dardenne, A. Le Bras, E. Marchand, and M. Marchal, "Real-time target tracking of soft tissues in 3d ultrasound images based on robust visual information and mechanical simulation," Medical Image Analysis, vol. 35, pp. 582-598, Jan 2017.

[7] H. T. Sen, N. Deshmukh, R. Goldman, P. Kazanzides, R. H. Taylor, E. Boctor, and N. Simaan, "Enabling technologies for natural orifice transluminal endoscopic surgery (N.O.T.E.S) using robotically guided elasticity imaging," in Medical Imaging: Image-Guided Procedures, Robotic Interventions, and Modeling, vol. 8316. SPIE, Feb 2012, pp. $83161 \mathrm{Y}-83161 \mathrm{Y}-8$.

[8] S. Billings, N. Deshmukh, H. J. Kang, R. Taylor, and E. M. Boctor, "System for robot-assisted real-time laparoscopic ultrasound elastography," in Medical Imaging: Image-Guided Procedures, Robotic Interventions, and Modeling, vol. 8316. SPIE, Feb 2012, pp. $83161 \mathrm{~W}-83161 \mathrm{~W}-8$

[9] C. Schneider, A. Baghani, R. Rohling, and S. Salcudean, "Remote ultrasound palpation for robotic interventions using absolute elastography," in Medical Image Computing and Computer-Assisted Intervention, Nice, 2012, pp. 42-49.

[10] R. Richa, R. Sznitman, R. Taylor, and G. Hager, "Visual tracking using the sum of conditional variance," in IEEE/RSJ Int. Conf. on Intelligent Robots and Systems, San Francisco, 2011, pp. 2953-2958.

[11] S. Baker and I. Matthews, "Lucas-kanade 20 years on: A unifying framework," International Journal of Computer Vision, vol. 56, no. 3, pp. 221-255, Feb 2004.

[12] B. Delabarre and E. Marchand, "Dense non-rigid visual tracking with a robust similarity function," in IEEE Int. Conf. on Image Processing, Paris, 2014, pp. 4942-4946.

[13] F. Brunet, V. Gay-Bellile, A. Bartoli, N. Navab, and R. Malgouyres, "Feature-driven direct non-rigid image registration," International Journal of Computer Vision, vol. 93, no. 1, pp. 33-52, Dec 2011.

[14] A. Krupa, G. Fichtinger, and G. Hager, "Real-time tissue tracking with b-mode ultrasound using speckle and visual servoing," in Int. Conf. on Medical Image Computing and Computer-Assisted Intervention, ser. LNCS 4792, N. Ayache, S. Ourselin, and A. Maeder, Eds., vol. 2, Brisbane, Oct. 2007, pp. 1-8.

[15] C. Nadeau and A. Krupa, "Intensity-based direct visual servoing of an ultrasound probe," in IEEE Int. Conf. on Robotics and Automation, Shanghai, 2011, pp. 5677-5682.

[16] B. Siciliano and J.-J. Slotine, "A general framework for managing multiple tasks in highly redundant robotic systems," in Fifth International Conference on Advanced Robotics., 1991, pp. 1211-1216.

[17] F. Kallel and J. Ophir, "A least-squares strain estimator for elastography," Ultrason Imaging, vol. 19, no. 3, pp. 195-208, Jul 1997.

[18] E. Marchand, F. Spindler, and F. Chaumette, "Visp for visual servoing: a generic software platform with a wide class of robot control skills," IEEE Robotics and Automation Magazine, vol. 12, no. 4, pp. 40-52, Dec 2005. 